\title{
Super Resolution methods to achieve high quality imaging
}

\author{
Prof.Bhushan V.Patil \\ R.C.Patel Institute of Technology, \\ Shirpur
}

\author{
Mayuri D.Patil \\ R.C.Patel Institute of Technology, \\ Shirbur \\ Dipali A. Shaha \\ R.C.Patel Institute of Technology, \\ Shirpur
}

\author{
Namrata R.Shaha \\ R.C.Patel Institute of Technology, \\ Shirpur
}

\begin{abstract}
Now a day the increase in the quality of images is becoming need of the multimedia applications. The quality of the image can be improved by increasing timage captured by any presentimage acquisition device. The increase in the optical resolution is having problem of shot noise due to smaller size of the sensors. Super Resolution (SR) deals with combining low resolution images of same scene with additional information in each to get higher resolution image. Due to this, we need not have a high resolution device for acquisition of an image. We present an overview of existing SR methods and address the motion estimation and optical blur methods of super resolution to achieve high quality imaging with different optical zoom of low resolution camera. The quality of images is significantly improved and high resolution imaging is achieved. These techniques are highly computational; but provide very good visual quality of images.
\end{abstract}

The full text of the article is not available in the cache. Kindly refer the IJCA digital library at www.ijcaonline.org for the complete article. In case, you face problems while downloading the full-text, please send a mail to editor at editor@ijcaonline.org 